\title{
Stewart-Treves syndrome: Magnetic resonance imaging data compared with pathological results from a single center
}

\author{
BIN LI and ZHENCHANG WANG
}

\author{
Department of Radiology, Beijing Friendship Hospital, Capital Medical University, Beijing 100050, P.R. China
}

Received November 24, 2015; Accepted May 4, 2017

DOI: $10.3892 / \mathrm{ol} .2017 .7363$

\begin{abstract}
The aim of the present study was to evaluate the magnetic resonance imaging (MRI) data of patients with Stewart-Treves Syndrome (STS), and to compare them with the corresponding pathological data. A total of 4,289 cases of secondary upper limb lymphedema (LE) from a single center, including 5 female cases of STS who underwent modified-radical post mastectomy, were included in the study. All cases were diagnosed pathologically, and by conventional and contrast-enhanced (ce)MRI scans. A total of 43 tumor nodules $(\Phi \geq 4 \mathrm{~mm}$ ) were examined. According to the immunohistochemical results, the 5 cases of STS were divided into 2 types; 4 patients exhibited STS type I [angiosarcoma (AS)] and 1 patient exhibited STS type 2 [mixed lymphangiosarcoma (mLAS)]. The MRI signal intensity (SI) of all the nodules was compared with the pathological results. In the T1WI sequences, all nodules showed isointensity compared with normal muscle tissues in the same image. However, in the T2WI sequences, there were the visually recognizable differences in the SI compared with the LE tissues in the two STS types. The SI of mLAS was more markedly decreased compared with that of AS. The SI of mLAS was closer to that of normal muscle tissues compared with the SI of AS. In the ceMRI, the SI of the tumor nodules was markedly increased in the two STS types, compared with non-enhancement imaging. Overall, MRI is a useful tool for the evaluation and diagnosis of STS. A chronic case of LE, in which MRI examination demonstrates nodules within the LE, may suggest a diagnosis of STS.
\end{abstract}

\section{Introduction}

Stewart-Treves syndrome (STS) is a rare but fatal tumor that has been associated with lymphedema (LE), with a 5-year survival rate of 5-35\% and a median survival time of 2.5 years (1-6).

Correspondence to: Dr Zhenchang Wang, Department of Radiology, Beijing Friendship Hospital, Capital Medical University, 95 Yong'an Road, Xicheng, Beijing 100050, P.R. China

E-mail: zcwang_vip@sina.com

Key words: chronic lymphedema, angiosarcoma, lymphangiosarcoma, Stewart-Treves syndrome, magnetic resonance imaging
If patients refuse treatment, the survival time is reduced to 5-8 months. Although STS exhibits a poor prognosis, an early diagnosis and prompt surgery are considered to be the main methods of prolonging survival $(7,8)$. Typically, chronic extremity LE leads to cutaneous hyperkeratosis that may mask the symptoms of early tumors involving the skin, but STS may also be mistaken for limb LE caused by exogenous factors, such as trauma (9). STS is also difficult to diagnose as invasive tumors almost always exhibit the appearance of benign tumors on magnetic resonance imaging (MRI), and this often leads to a delayed diagnosis. In addition, it has been hypothesized that STS exhibits multifocal nodules and a tendency for early metastasis (10), but, to the best of our knowledge, no studies have validated this. Studies on the use of MRI in STS are limited, due to the costs of this technique. In previous studies $(8,9)$, STS were observed by MRI; however, no information was provided regarding cluster of differentiation 31 (CD31) and D2-40. In the present study, clinical data on 5 STS cases was collected, including MRI, light microscopy examination and immunohistochemical results (including CD31 and D2-40) $(8,9)$. Although, the diagnostic term 'STS' was first used in 1948 and the pathological term 'lymphangiosarcoma' (LAS) was initially used in the same study (1). Subsequently, angiosarcoma (AS) and LAS were typically used interchangeably, and applied and interpreted as a synonymous diagnostic term $(8,11,12)$.

The present study aimed at analyzing the MRI data of AS and compare them with the pathological results of STS, in order to provide a tool that may aid the diagnosis of the syndrome.

\section{Patients and methods}

Patients. Between July 2008 and March 2015, a total of 4,289 female cases (age range, 42-78 years; mean \pm standard deviation, $60.6 \pm 16.5$ years) of secondary upper limb LE that were treated at a single center (Beijing Friendship Hospital, Beijing, China) were included in the present study. Of the studied population, 5 women (range, 56-78 years; median, 65 years) with a diagnosis of STS who underwent modified-radical post mastectomy followed by radio-chemotherapy, and whose diagnosis was confirmed pathologically, were included in the analysis. These accounted for $0.12 \%$ of the total study population. The patients' ages ranged between 56 and 78 years, with a median age of 62 years. The range of duration of LE was 9-17 years, with a median duration of 13 years. A 
total of 4 patients with STS had no obvious pathogenic causes and 1 patient presented with a history of trauma in the elbow joint that occurred 2 months previously. A total of 2 patients presented with STS in the dermis. The patients were treated by wide excision and exhibited a survival time of $>15$ and $>18$ months, respectively. The other 3 patients presented with STS located in the dermis and subcutaneous tissue (s-ct) which was defined from the sub-dermis to the surface of muscle. The 2 patients with STS type I (AS) were treated by disarticulation and exhibited short survival times (6 and 9 months, respectively). The 1 patient with STS type II [mixed LAS (mLAS)] was treated by amputation, including the elbow joint, and exhibited a longer survival time of $>24$ months (Table I). The study protocol was approved by Beijing Friendship Hospital (Beijing, China).

Instruments and methods. The GE Signa 1.5T MRI unit (GE Healthcare, Chicago, IL, USA) with a 16-channel body surface coil was the MRI imaging system used. The regions of interest of the limb LE were placed into the center coil as possible. The conventional MRI, including T1-weighted image (WI)/axial image (AXI) used a repetition time (TR) of $500 \mathrm{msec}$ and an echo time (TE) of $15 \mathrm{msec}$, with a slice thickness of $8 \mathrm{~mm}$ and a slice gap of $0.8 \mathrm{~mm}$. The field of view (FOV) was $38 \times 30 \mathrm{~cm}$, the matrix was $320 \times 224$ and the number of excitations (NEX) was 2. The T2WI/AXI use a TR of 3,600 msec/TE $110 \mathrm{msec}$, with a slice thickness of $8 \mathrm{~mm}$ and a slice gap of $0.8 \mathrm{~mm}$. The FOV was $38 \times 30 \mathrm{~cm}$, the matrix was $384 \times 288$ and the NEX was 2 . The short TI inversion recovery (STIR)/AXI used a TR of 4,000 $\mathrm{msec} / \mathrm{TE} 110 \mathrm{msec} /$ time inversion recovery $155 \mathrm{msec}$, with a slice thickness of $8 \mathrm{~mm}$ and a slice gap of $0.8 \mathrm{~mm}$. The FOV was $40 \times 40 / 38 \times 30 \mathrm{~cm}$, the matrix was $320 \times 224$ and the NEX was 4 . The STIR sequence was selectable (only in patients in a good condition). All cases underwent contrast-enhanced (ce)MRI; the contrast agent, gadopentetic acid (Consun Pharmaceutical Co., Ltd., Guangzhou, China), was injected through the median cubital vein, at a dose of $0.1 \mathrm{mmol} / \mathrm{kg}$ body weight, using a high pressure syringe at a flow rate of $2.0 \mathrm{ml} / \mathrm{sec}$. All patients provided written informed consent.

The 5 patients with STS were randomly assigned to 2 senior radiologists. The location, number, outline and signal intensity of nodules ( $\Phi \geq 4 \mathrm{~mm}$ ) were screened and analyzed by conventional MRI and ceMRI. If the two radiologists held a different view about any nodule, it was excluded. The ceMRI facilitated the diagnosis of infiltrative and confluent growth. As the fiber cable in LE and the deep fascia demonstrate a line shape, if the fiber cable and/or deep fascia became abnormally thickened and enhanced beside the nodule, these signs indicated that the nodules exhibited significant infiltrative growth. If the outline of the nodules was irregular and visible as a notch appearance, it indicated a confluent nodule.

The removed surgical specimens were fixed in $10 \%$ formalin within $12 \mathrm{~h}$ of collection and embedded in paraffin for light microscopy examination (magnification, x200). The tissue sections (thickness, $6 \mu \mathrm{m}$ ) were stained with hematoxylin and eosin (H\&E; Zhongshan Biotechnology, Guangdong Province, China), as described previously $(8,12)$. The immunohistochemical staining processes of the pathological specimens were performed using a Dako Automated Staining instrument (cat. no. ZYB/USA2016-2012; Dako; Agilent Technologies, Inc., Santa Clara, CA, USA) according to the manufacturer's protocol. All immunohistochemical processes were performed at $37^{\circ} \mathrm{C}$. Subsequently, according to the expression of the endothelial marker CD31 (anti-CD31; cat. no. JC/70A; dilution, 1:80; Dako; Agilent Technologies, Inc.) and lymphatic marker D2-40 (anti-D2-40; cat. no. M3619; dilution, 1:60; Dako; Agilent Technologies, Inc.), the cases were divided into the following 3 types: STS type I, strongly positive for CD31 and weakly positive for D2-40; STS type II 1 (pure LAS; pLAS), strongly positive for D2-40 and weakly positive for CD31; and STS type II 2 (mLAS), moderately positive for D2-40 and CD31. The analysis of expression in immunohistochemical results was performed by two experienced pathologists. If disagreement between the two pathologists occurred, after reaching the consensus, the results of analysis were included in this study.

\section{Results}

Pathological types. STS types I and II are presented in Figs. 1 and 2, respectively. In the STS I type, including 37 nodules (Tables II and III), the H\&E staining revealed a densely hypercellular tumor and the tendency of infiltration (Fig. 1A). The expression of CD31 was positive (Fig. 1B) and the expression of D2-40 was negative (Fig. 1C) or weakly positive (Table II). No nodules of STS type II 1 were included in this study. In STS type II 2 including 6 nodules (Tables II and III), H\&E staining revealed more densely hypercellular tumor than the STS I type, and lack of vessels (Fig. 2A). The expression of CD31 was positive (Fig. 2B), as was the expression of D2-40 (Fig. 2C), which was classified as STS type II 2 (mLAS). The expression indices of Ki-67 and cellular tumor antigen p53 (p53) were also detected. (Table II).

MRI data. A total of 43 nodules were studied, of which 16 nodules were located in the dermis, and 27 nodules in the s-ct. The 2 patients with AS exhibited tumors that were located in the dermis, with 5 nodules. Of the 3 patients with tumors of mixed location, 2 patients with AS presented with 32 nodules ( 9 nodules in the dermis and 23 nodules in the s-ct) (Fig. 1D-F) and 1 patient with mLAS presented with 7 nodules ( 2 nodules in the dermis and 5 nodules in the s-ct). In the patient with mLAS, there was a triangular region (Fig. 2D and E) with slightly low signal intensity (SI) compared with the muscle signals, in the lateral dermis of the elbow. But in the ceMRI, 2 enhanced nodules in the triangular region were detected (Fig. 2F). Therefore, the 2 nodules were included into the total count of dermis nodules.

Outline of nodules. As the nodules in the dermis exhibited an appearance of fusion growth and cutaneous hyperkeratosis, their outline was not satisfactorily detected in the T2WI sequence. However, in ceMRI, the outline of all 16 nodules was determined satisfactorily. The nodules in the s-ct demonstrated a clear outline against LE in the T2WI scans (Figs. 1E and $2 \mathrm{H}$ ). Of all the 27 nodules in the s-ct, 23 nodules exhibited the appearance of infiltrative growth, with abnormally thickened fiber cables and/or deep fascia. There were 2 confluent nodules in the patient with AS and 1 confluent nodule in 
Table I. Clinical data and surgical history in patients with Stewart-Treves syndrome.

\begin{tabular}{lllll}
\hline Patient & $\begin{array}{c}\text { Age, } \\
\text { years }\end{array}$ & \multicolumn{1}{c}{$\begin{array}{c}\text { Prior } \\
\text { treatment }\end{array}$} & $\begin{array}{c}\text { Duration of } \\
\text { lymphedema, years }\end{array}$ & $\begin{array}{c}\text { Survival } \\
\text { Surgery } \\
\text { time, months }\end{array}$ \\
\hline 1 & 78 & Mastectomy and radio-chemotherapy & 17 & Disarticulation \\
2 & 60 & Mastectomy and radio-chemotherapy & 11 & Disarticulation \\
3 & 65 & Mastectomy and radio-chemotherapy & 10 & Wide excision \\
4 & 62 & Mastectomy and radio-chemotherapy & 13 & Wide excision \\
5 & 56 & Mastectomy and radio-chemotherapy & 14 & Amputation \\
\hline
\end{tabular}

Table II. Results of immunohistochemistry and pathological types in patients with STS.

\begin{tabular}{|c|c|c|c|c|c|}
\hline \multirow[b]{2}{*}{ Cell marker } & \multicolumn{4}{|c|}{ STS type I } & \multirow{2}{*}{$\frac{\text { STS type II }}{\text { Patient } 5}$} \\
\hline & Patient 1 & Patient 2 & Patient 3 & Patient 4 & \\
\hline CD31 & + & + & + & + & + \\
\hline D2-40 & - & - & \pm & - & + \\
\hline $\mathrm{Ki}-67, \%$ & $90-95$ & 60 & 20 & 50 & 30 \\
\hline p53 & $t^{\mathrm{a}}$ & $t^{\mathrm{a}}$ & - & $\pm^{\mathrm{a}}$ & - \\
\hline
\end{tabular}

STS, Stewart-Treves syndrome; -, negative; \pm , weakly positive; + , positive; $\pm^{\mathrm{a}}$, individual-cell positive; $+^{\mathrm{a}}$, multifocal positive.

Table III. Magnetic resonance imaging data in patients with Stewart-Treves syndrome.

\begin{tabular}{|c|c|c|c|c|}
\hline Patient no. & Location of nodule & Number of nodules, dermis/s-ct & $\mathrm{T} 2 \mathrm{WI}^{\mathrm{a}}$ & $\mathrm{T} 2 \mathrm{WI}^{\mathrm{b}}$ \\
\hline 1 & Forearm; dermis/s-ct ${ }^{\mathrm{c}}$ & $5 / 15$ & Slightly decreased & Higher \\
\hline 2 & Upper arm; dermis/s-ct ${ }^{\mathrm{c}}$ & $4 / 8$ & Slightly decreased & Higher \\
\hline 3 & Upper arm; dermis & $3 / 0$ & Slightly decreased & Higher \\
\hline 4 & Forearm; dermis & $2 / 0$ & Slightly decreased & Higher \\
\hline 5 & $\begin{array}{l}\text { Lateral elbow and forearm; } \\
\text { dermis/s-ctc }\end{array}$ & $2 / 4$ & Markedly decreased & $\begin{array}{l}\text { Slightly } \\
\text { higher }\end{array}$ \\
\hline
\end{tabular}

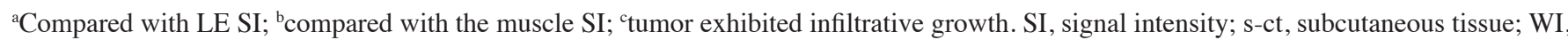
weighted imaging.

the patient with mLAS. These confluent nodules exhibited abnormally thickened and enhanced close fiber cables and deep fascia (Figs. 1F, 2F and 2I). There were 13 non-confluent nodules; 10 of these only exhibited abnormally thickened close fiber cables, while the other 3 only exhibited abnormally thickened close deep fascia. The diameters of 13 non-confluent nodules ranged between $5-8 \mathrm{~mm}$. The mean diameter of the 4 nodules without infiltrative growth or fusion was $4 \mathrm{~mm}$.

SI. The nodules of the 2 STS types were isointense compared with the normal muscle in the same sections in the T1WI sequences, yet there was no marked signal difference between the 2 STS types (Figs. 1D, 2D and 2G). Their SI was decreased by various degrees compared with LE in T2WI sequence, but they demonstrated a visually recognizable signal difference. The SI in the patients with mLAS was more markedly decreased (Fig. 2E and $2 \mathrm{H}$ ) compared with that of the patients with AS (Fig. 1E). When comparing the SI of the normal muscle to the tumor nodules in the same slice, the SI of mLAS was closer to that of the muscle compared with the SI of AS. The SI of AS was slightly decreased even in comparison with LE, and was higher compared with that of muscle. The nodules were markedly enhanced following intravenous contrast in the 2 types of STS (Table III).

\section{Discussion}

Chronic LE is an essential and important aspect of the pathogenesis of STS (1-7). There are numerous factors that contribute to this pathogenesis, including but not limited to tumor radical surgery (radical mastectomy or radical hysterectomy), lymph node excision, trauma and inflammation. However, there are also cases of idiopathic LE (8-12). The identified patients with STS involving the upper limb following radical mastectomy 

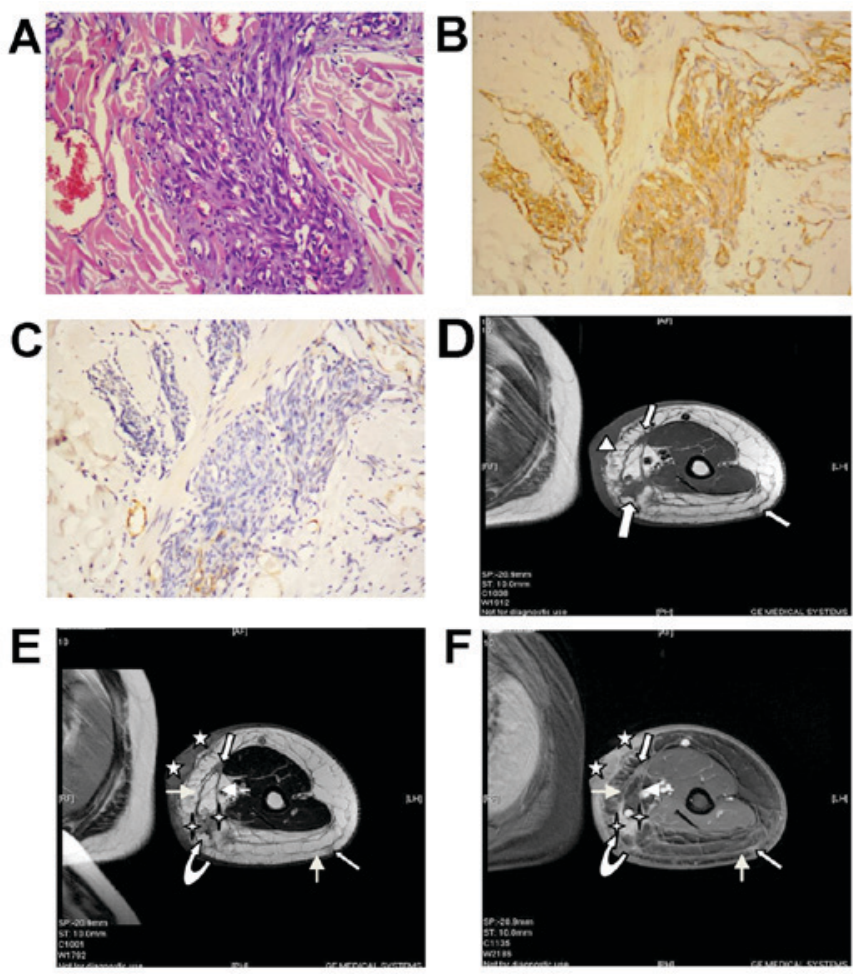

Figure 1. Angiosarcoma (STS-I type). (A) Hematoxylin and eosin histopathology at original magnification $\times 200$ revealed that the tumor was composed predominantly of atypical epithelioid cells with a moderate degree of nuclear atypia, and also exhibited invasive growth. There were a high number of immature vessels diffused throughout the tumor and small mature vessels in collagen fiber with red blood cells. (B) Cluster of differentiation 31 staining was positive (magnification, $\mathrm{x} 200$ ). (C) D2-40 staining was negative (magnification, $\mathrm{x} 200$ ). (D) Axial T1WI demonstrates skin thickening in a large range of the patient's limb with regional processes (indicated by triangle). It indicates at least three nodules (indicated by outlined arrows) in the s-ct which was defined from the subdermis to the surface of muscle, with markedly thickened adjacent fibers. Also noted is the extensive circumference of the upper arm, consistent with LE. (E) Corresponding axial FSE T2WI demonstrates more tumor nodules with well-defined outlines. Scans indicate that the SI of the 2 hidden dermal nodules (indicated by stars) and 4 subcutaneous nodules is decreased slightly compared with the LE tissue, and is higher compared with that of the underlying muscle with normal SI. The larger nodule (indicated by curved arrow) in the s-ct is 1 nodule made up of two fused smaller nodules (indicated by 4-pointed star), and the outline is irregular with the appearance of visible incisura with markedly thickened adjacent fibers (indicated by plain arrows). The outlined white arrows indicate tumor nodules in the s-ct. The deep thickened fascia between the 2 nodules was observed. The above signs indicate that the tumor is exhibiting invasive growth. The extensive LE was apparent. (F) Corresponding axial post-gadolinium T1WI demonstrates diffuse contrast enhancement within the tumor nodules. Adjacent thickened skin is markedly enhanced. The 5-pointed stars indicate the 2 hidden dermal nodules. The 4-pointed stars indicate the two fused smaller nodules in the s-ct. The curved outline arrow indicates the larger nodule in the s-ct. The plain white arrows indicate thickened adjacent fibers. The outlined white arrows indicate the tumor nodules in the s-ct. LE, lymphedema; WI, weighted image; SI, signal intensity; s-ct, subcutaneous tissue; FSE, fast spin echo.

with or without radiotherapy account for $\sim 90 \%$ of the total cases described $(6,11)$. Nascimento et al (12) hypothesized that the incidence of STS has markedly decreased due to the improvements in surgery and radiation therapy techniques, and the popularity of breast conservation therapy. The incidence of STS in the present study was $0.12 \%$ and was therefore consistent with previous studies $(2,6)$. The duration of LE and the age of STS onset were consistent with those suggested in the literature $(2,3)$. There was 1 patient with impact trauma 2 months previously, in whom nodules in the dermis and s-ct were detected using ceMRI. This appearance was described in previous studies $(9,10)$, and therefore it was not discussed. It was not considered possible that the impact trauma was the direct causal factor of STS, despite of the 2 nodules in the dermis. It is possible that following a trauma in the fragile region of $\mathrm{LE}$, it becomes difficult to repair the local damage, and that a state of immunodeficiency would then occur gradually and latently in this region, ultimately leading to STS. It was reasoned that the 4 cases of STS without apparent causes were closely associated with the occurrence of immunodeficiency in LE (12-16).

In 1948, Stewart and Treves (1) first used the term LAS in a pathological setting. In the following decades, AS and LAS were often used interchangeably and applied and interpreted as a diagnostic term synonymous for angiosarcoma, until 2011 when Yu and Yang (17) clearly explained the pathological diagnosis standards of pLAS, and determined that AS and LAS were actually misnomers. D2-40 is an available selective marker for the lymphatic endothelium, and the sensitivity and the positive predictive value of the diagnosis of LAS were shown to be excellent (18-20). CD31 is an adequate and approved marker for vascular endothelial markers in long-term clinical application (21), and it is able to identify tumor cells derived from the vascular endothelial cells. In a study investigating pLAS (17), CD31 was weakly positive or negative, and D2-40 was positive. While in the present study, CD31 was positive in all 5 cases, this meant that no cases were classed as pLAS, but each of the tumors consisted of vascular endothelial tumor cells. In total, 1 out of 5 patients exhibited positive expression of D2-40. The present study followed previous advice (17) that all tumors that express a mixed immunophenotype of CD31 and D2-40 should be classified as a subset of LAS. It has been previously suggested that tumor cells demonstrate at least partial differentiation along the lymphatic endothelial lineage (18-20). As such, the present patient, who demonstrated a mixed immunophenotype, was diagnosed with mLAS. The other 4 cases were classified as AS.

It was observed that the tumors demonstrated a visually recognizable signal difference in the T2WI scans in the 2 STS types. The SI of AS and LAS was decreased compared with that of LE, but the SI of mLAS was decreased more markedly compared with that of AS. If compared with the normal muscle tissues, the SI of mLAS was closer to that of muscle tissues compared with that of AS, and the SI of AS was higher compared with that of muscle tissue. Through analyzing and comparing MRI signals with the histopathological results, it was identified that the tumors were composed predominantly of dense atypical cells with oval nuclear contours, prominent nucleoli and eosinophilic cytoplasm in the 2 STS types (Figs. 1A and 2A). In the AS tissues, the collagen fibrils were abundantly distributed between the tumor nests. The structure of dense tumor cells with or without collagen fibrils is the basis of the tumor entity. In certain areas of the mLAS tumors, specific clefts and slit-like channels were identified, occasionally with specific sections that contained proteinaceous fluid and a small number of lymphocytes (Fig. 2A). Conversely, in the AS tumors, numerous clefts and channels were present and were markedly dilated, and always contained free erythrocytes. 

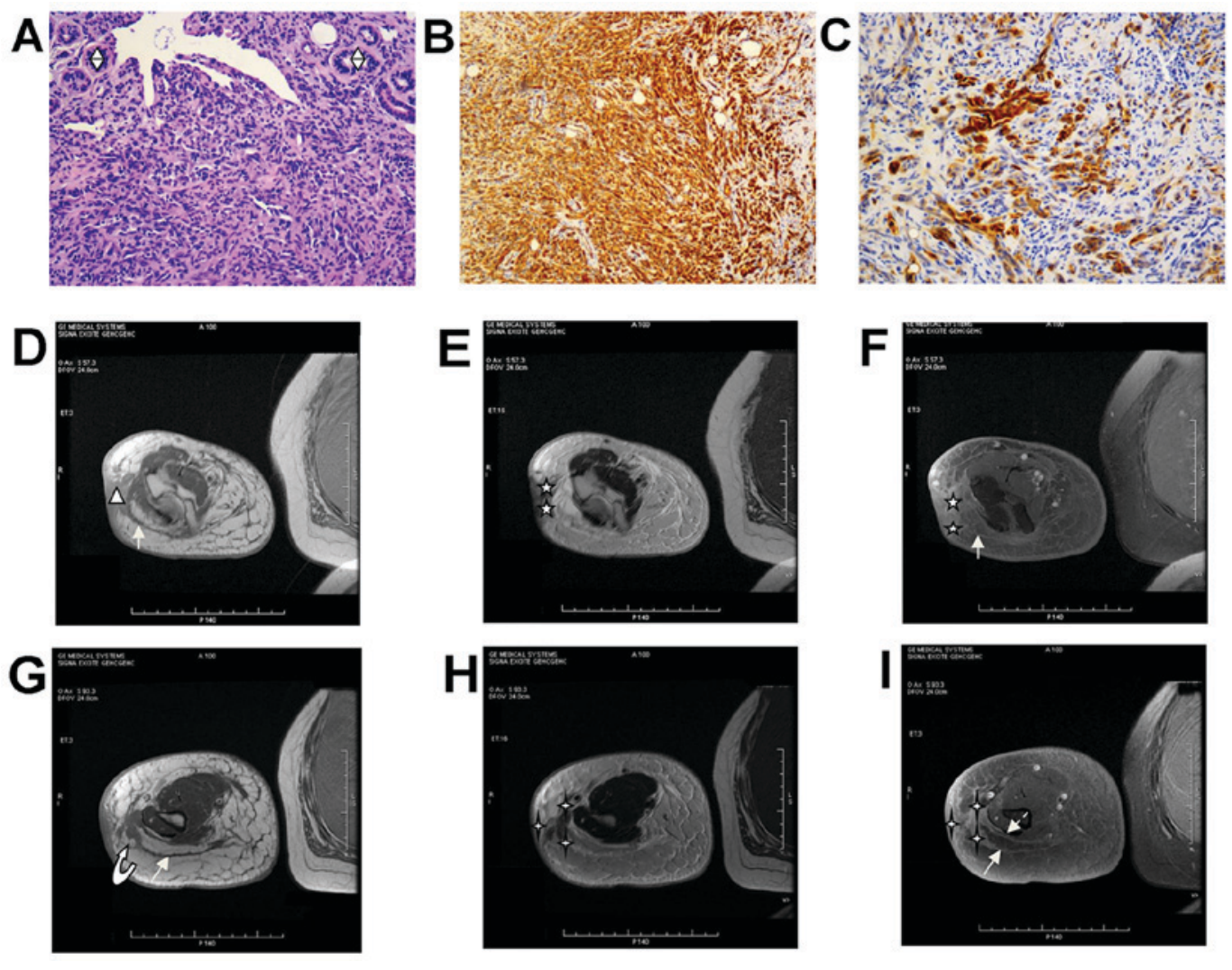

Figure 2. Lymphangiosarcoma (STS-II 2 type). (A) Hematoxylin and eosin histopathology (magnification, x200) revealed a compact tumor with invasive growth that was composed of densely atypical epithelioid cells. The tumor cells exhibit a moderate degree of nuclear atypia. There are a lack of vessels. In certain areas, these atypical epithelioid cells formed clefts and numerous, anastomosing or markedly dilated, slit-like erythrocyte-free channels that occasionally contained proteinaceous fluid and a small number of lymphocytes. Skin adnexal structures were visible (indicated by split diamonds). (B) Cluster of differentiation 31 staining was positive (magnification, x200). (C) D2-40 staining was partially positive (magnification, x200). (D) Axial T1WI demonstrates the focal thickening skin with an irregular outline (triangle), and with extension into s-ct by $9 \mathrm{~mm}$. It demonstrates markedly thickened adjacent fibers (plain white arrow), with a burr-like projection. (E) Corresponding axial FSE T2WI demonstrates that that the 2 tumor nodules (indicated by stars) are hidden in the triangle-shape region of the signal, in which the SI of tumor nodules is markedly decreased compared with the LE tissues, and is closer to the SI of the underlying muscle tissue. The outlines are relatively well-defined. (F) Corresponding axial post-gadolinium T1WI demonstrates contrast enhancement in the tumor nodules, and the adjacent thickened skin is more enhanced compared with the tumor nodules. The 5-pointed angle stars indicate the 2 hidden dermal nodules. The plain white arrows indicate thickened adjacent fibers. (G) Axial T1WI demonstrates 3 tumor nodules with fusion growth (indicated by curved arrow), which exhibits the appearance of an incisura in the subcutaneous tissue. It also demonstrates markedly thickened adjacent cable-like shapes (indicated by plain arrow) beside the tumor. (H) Corresponding axial FSE T2WI demonstrates that the SI of the 3 tumor nodules (indicated by 4-pointed stars) was markedly decreased compared with the LE tissue, and is closer to the SI of the underlying muscle. The outlines are well-defined. (I) Corresponding axial post-gadolinium T1WI demonstrates contrast enhancement in the tumor nodules, and the adjacent and involved thickened fibers are also enhanced. The thickened deep fascia (indicated by plain arrows) on the muscle surface is involved and markedly enhanced. The 4-pointed stars indicate the 3 tumor nodules in the s-ct. These results did not confirm the signs demonstrated in the conventional MRI scan. WI, weighted image; signal intensity; FSE, fast spin echo; s-ct, subcutaneous tissue.

It was also observed that in the AS tumors there were a number of immature vessels in the tumor nests and mature vessels in the collagen fibrils (Fig. 1A). The large number of vascular vessels may suggest that there was an increased proportion of water molecules in the AS tumors. However, the mLAS tumors clearly lacked these. It was hypothesized that the overall structure in all types of tumors caused the SI of the tumor to be decreased in comparison with LE areas. The different proportions of vascular vessels caused the changes in SI by various degrees compared with either normal muscle or LE tissues in the T2WI sequences. A potential reason for this is that the tumors demonstrated a visually recognizable difference in signals in the two STS types. It is hypothesized that there are two reasons why there are SI alterations in MRIs: One is the proportion of tumor cells and blood vessels in the tumor $(22,23)$, and the other is due to the abnormal amount of fat and fibrous tissues in LE (23). A similar difference occurred in the present study too. Therefore, it is suggested that the SI of normal muscle should be used as the internal reference, in order to reduce the random subjective visual interference and to obtain a unified idea of understanding of the SI.

Although there is trend and potential for multifocal growth and early transformation in STS, confirmatory studies are extremely rare (10). According to current knowledge, the MRI data concerning these factors have not been described. In the present study, there were not only 3 cases of multifocal distribution, but also 13 nodules and 3 confluent nodules with a diameter of $>5 \mathrm{~mm}$ that exhibited a clear infiltration growth trend in the s-ct. There were greater numbers of transferred nodules ( 23 nodules) in the 2 patients with AS in the s-ct compared with the numbers in the patient with mLAS (4 nodules). This was hypothesized to be associated with the existing vascular lumen structure in the patients with AS and that the vascular vessels provided blood supply for the growth of tumor and establish tumor transfer along the vascular luminal structure, acting as a 'highway'. However, 1 mLAS 
case exhibited a lack of a vascular lumen structure. The expression Ki-67 and p53 indices are closely associated with the survival time; as suggested by the 2 patients with AS and 1 patient with mLAS. The expression $\mathrm{Ki}-67$ and p53 indices in the patients with AS were markedly higher compared with that of the patient with mLAS, but the survival time demonstrated the opposite outcome. This difference indicates that the degree of malignancy of AS with no clear cause is higher compared with that of mLAS with exogenous causal factors, such as trauma, and also suggested the hypothesis that oncogenesis is closely associated with the region of focal immunodeficiency in LE (12-16). The reasons were as follows: Although the expression of $\mathrm{Ki}-67$ and $\mathrm{p} 53$ was not mentioned in the previous studies investigating STS, it was hypothesized that the expression of each index was actually associated with prognosis, not with age. There were two unavoidable limitations to the present study namely that the observation time was short and that the number of enrolled cases was only 5 .

In conclusion, the data of the present study suggest that: i) Although it is difficult to clinically estimate the degree of focal immunodeficiency in LE, it is feasible to perform MRI scans for patients who have exhibited LE for $>10$ years. It is necessary for patients to avoid trauma in the limb with LE to reduce the number of patients with STS.ii) For the patients with STS only located in the dermis, it may be beneficial to perform a wide excision surgery, which may have a good prognosis. For multifocal STS with early metastasis, the prognosis of LAS may be improved compared with that of AS. If the number of metastatic nodules in the s-ct is $>8$ in AS, even amputation may result in a poor prognostic outcome. iii) In conventional MRI scans, it is easy to detect a visually recognizable signal difference between the two STS types compared with histological examination. Obtaining a unified understanding of the tumor SI by use of the normal muscle and LE SIs as references will also assist this. The MRI scans provide a more accurate evaluation of multifocality and stages of early transformation.

It is hypothesized that MRI scans are a useful tool for evaluating STS, and that they also provide important diagnostic information in a clinical setting. A chronic case of LE, in which MRI scans demonstrate the nodules in the LE, suggest a diagnosis of STS.

\section{References}

1. Stewart FW and Treves N: Lymphangiosarcoma in postmastectomy lymphedema; a report of six cases in elephantiasis chirurgica. Cancer 1: 64-81, 1948.

2. Fitzpatrick PJ: Lymphangiosarcoma and breast cancer. Can J Surg 12: 172-177, 1969.
3. Maldonado-Fernández N, López-Espada C, Sánchez-Rodriguez J, Rodríguez-Morata A, Fernández-Quesada F, Martínez-Gámez J, Moreno-Escobar J and García-Róspide V: Síndrome de Stewart-Treves: Linfangiosarcoma en linfedema crónico posmastectomía. Angiología 54: 467-471, 2002.

4. Young RJ, Brown NJ, Reed MW, Hughes D and Woll PJ: Angiosarcoma. Lancet Oncol 11: 983-991, 2010.

5. Sordillo PP, Chapman R, Hajdu SI, Magill GB and Golbey RB: Lymphangiosarcoma. Cancer 48: 1674-1679, 1981.

6. Woodward AH, Ivins JC and Soule EH: Lymphangiosarcoma arising in chronic lymphedematous extremities. Cancer 30: 562-572,1972.

7. Grobmyer SR, Daly JM, Glotzbach RE and Grobmyer AJ III: Role of surgery in the management of postmastectomy extremity angiosarcoma (Stewart-Treves syndrome). J Surg Oncol 73: 182-188, 2000.

8. Kerchner K, Fleischer A and Yosipovitch G: Lower extremity lymphedema update: Pathophysiology, diagnosis, and treatment guidelines. J Am Acad Dermatol 59: 324-331, 2008.

9. Borel Rinkes IH and de Jongste AB: Lymphangiosarcoma in chronic lymphedema. Reports of 3 cases and review of the literature. Acta Chir Scand 152: 227-230, 1986.

10. Peyron N, Dandurand M and Guillot B: Malignant tumors as complications of lymphedema. J Mal Vasc 18: 293-298, 1993 (In French).

11. Martin MB, Kon ND, Kawamoto EH, Myers RT and Sterchi JM: Postmastectomy angiosarcoma. Am Surg 50: 541-545, 1984.

12. Nascimento AF, Raut CP and Fletcher CD: Primary angiosarcoma of the breast: Clinicopathologic analysis of 49 cases, suggesting that grade is not prognostic. Am J Surg Pathol 32: 1896-1904, 2008.

13. Futrell $\mathrm{J}$ and Meyers GH Jr: The regional lymphatics and cancer immunity. Ann Surg 177: 1-7, 1973.

14. Ruocco V, Brunetti G, Puca RV and Ruocco E: The immunocompromised district: A unifying concept for lymphoedematous, herpes-infected and otherwise damaged sites. J Eur Acad Dermatol Venereol 23: 1364-1373, 2009.

15. Ruocco V, Schwartz RA and Ruocco E: Lymphedema: An immunologically vulnerable site for development of neoplasms. J Am Acad Dermatol 47: 124-127, 2002.

16. Ruocco V, Ruocco E, Schwartz RA and Janniger CK: Kaposi sarcoma and quinine: A potentially overlooked triggering factor in millions of Africans. J Am Acad Dermatol 64: 434-436, 2011.

17. Yu L and Yang SJ: Lymphangiosarcoma of the vocal cord: A rare entity defined by a D2-40 immunohistochemical and ultrastructural study. J Clin Oncol 29: e57-e61, 2011.

18. Kahn HJ, Bailey D and Marks A: Monoclonal antibody D2-40, a new marker of lymphatic endothelium, reacts with Kaposi's sarcoma and a subset of angiosarcomas. Mod Pathol 15: 434-440, 2002.

19. Fukunaga M: Expression of D2-40 in lymphatic endothelium of normal tissues and in vascular tumours. Histopathology 46: 396-402, 2005.

20. Mankey CC, McHugh JB, Thomas DG and Lucas DR: Can lymphangiosarcoma be resurrected? A clinicopathological and immunohistochemical study of lymphatic differentiation in 49 angiosarcomas. Histopathology 56: 364-371, 2010.

21. Ohsawa M, Naka N, Tomita Y, Kawamori D, Kanno H and Aozasa K: Use of immunohistochemical procedures in diagnosing angiosarcoma. Evaluation of 98 cases. Cancer 75: 2867-2874, 1995.

22. Schindera ST, Streit M, Kaelin U, Stauffer E, Steinbach L and Anderson SE: Stewart-Treves syndrome: MR imaging of a postmastectomy upper-limb chronic lymphedema with angiosarcoma. Skeletal Radiol 34: 156-160, 2005.

23. Nakazono T, Kudo S, Matsuo Y, Matsubayashi R, Ehara S, Narisawa $\mathrm{H}$ and Yonemitsu N: Angiosarcoma associated with chronic lymphedema (Stewart-Treves syndrome) of the leg: MR imaging. Skeletal Radiol 29: 413-416, 2000. 